\title{
openheart Pacing-associated cardiomyopathy in adult congenital heart disease
}

\author{
Benjamin M Moore (1) ,1,2 Caroline Medi, ${ }^{1,2}$ Mark A McGuire, ${ }^{1,2}$ \\ David S Celermajer, ${ }^{1,2,3}$ Rachael L Cordina ${ }^{1,2,4}$
}

\begin{abstract}
- Additional material is published online only. To view please visit the journal online (http://dx.doi.org/10.1136/ openhrt-2020-001374).
\end{abstract}

To cite: Moore BM, Medi C, McGuire MA, et al. Pacingassociated cardiomyopathy in adult congenital heart disease. Open Heart 2020;7:e001374. doi:10.1136/

openhrt-2020-001374

Received 27 June 2020 Revised 26 October 2020 Accepted 1 December 2020

Check for updates

(c) Author(s) (or their employer(s)) 2020. Re-use permitted under CC BY-NC. No commercial re-use. See rights and permissions. Published by BMJ.

${ }^{1}$ Cardiology, Royal Prince Alfred Hospital, Camperdown, New South Wales, Australia

${ }^{2}$ Sydney Medical School, The University of Sydney, Camperdown, New South Wales, Australia

${ }^{3}$ Heart Research Institute, Newtown, NSW, Australia ${ }^{4}$ Murdoch Children's Research Institute, Parkville, Victoria, Australia

Correspondence to Dr Rachael L Cordina; rachcordina@yahoo.com

\begin{abstract}
Objectives Long-term single-site ventricular pacing may adversely affect ventricular function, due to dyssynchronous systemic ventricular contraction. We sought to determine the incidence, predictors and outcomes of pacing-associated cardiomyopathy (PACM) in an adult congenital heart disease (ACHD) cohort.

Methods We retrospectively identified all patients in our database with a permanent pacemaker from 2000 to 2019. Patients were followed for the primary endpoint of unexplained decline in systemic ventricular function (PACM) and the secondary endpoint of heart failure admission.
\end{abstract}

Results Of 2073 patients in our database, 106 had undergone pacemaker implantation. Over a median followup of 9.4 years, 25 patients (24\%) developed PACM, but only in those with ventricular pacing percentage (VP\%) $\geq 70 \%$; PACM occurred in $0 \%$ of those with VP $<70 \%$ and $47 \%$ of those with VP $\geq 70 \%$ ( $p<0.001)$. High-burden ventricular pacing $(\geq 70 \%)$ remained predictive of PACM in transposition of the great arteries, tetralogy of Fallot and complex biventricular repair subgroups, but not in Fontan patients. Those with PACM were more likely to be admitted with heart failure ( $44 \%$ vs $15 \%, p=0.002)$. Cardiac resynchronisation therapy (CRT) upgrade was performed in 11 patients, with 9 responders $(82 \%)$.

Conclusions In a cohort of patients with ACHD followed long-term post-pacing, 24\% developed cardiomyopathy that was significantly associated with a higher burden of ventricular pacing (VP $\geq 70 \%$ ). Given promising response rates to CRT, patients with ACHD expected to pace in the ventricle should be closely monitored for systemic ventricular decline.

\section{INTRODUCTION}

The potential adverse effects of chronic single-site ventricular pacing have been well described in acquired heart disease ${ }^{1-4}$ Persistent dyssynchronous left ventricular (LV) contraction, due to right ventricular pacing, may result in systolic LV impairment. ${ }^{5}$ This is termed 'pacing-induced cardiomyopathy', in the absence of another cause. ${ }^{34}$ The risk of clinical heart failure is also increased in these individuals. ${ }^{12}$ At a histopathological level, myocardial biopsies from young patients with congenital complete heart block (CHB) and chronic pacing have demonstrated

\section{Key questions}

What is already known about this subject?

- In acquired heart disease, long-term single-site ventricular pacing may result in pacing-induced cardiomyopathy and/or heart failure, in susceptible individuals. Patients with pre-existing ventricular impairment or high-burden ventricular pacing are at elevated risk. Patients with adult congenital heart disease (ACHD) may be more vulnerable to singlesite ventricular pacing, due to multiple underlying risk factors for heart failure.

What does this study add?

- We found one in four patients with ACHD followed long-term post-pacing developed an unexplained cardiomyopathy, but only in those with ventricular pacing $\geq 70 \%$. High-burden ventricular pacing $(\geq 70 \%)$ remained predictive of cardiomyopathy in transposition of the great arteries, tetralogy of Fallot and complex biventricular repair subgroups. Response rates to cardiac resynchronisation therapy (CRT) upgrade exceeded $80 \%$.

How might this impact on clinical practice?

- Surveillance echocardiography should be performed in patients with ACHD expected to pace even at a modest burden in the ventricle, given the positive CRT response rate in this population. Algorithms to reduce ventricular pacing percentage may be beneficial, although endpoints of this intervention in ACHD have not yet been assessed. Consideration should be given to de novo CRT implantation in 'atrisk' individuals with ACHD with a pacing indication.

degenerative LV fibrosis. ${ }^{6}$ The prevalence of significant bradycardia requiring pacing in patients with adult congenital heart disease (ACHD) substantially exceeds the age-equivalent general population. ${ }^{7}$ Pacemakers are most commonly dual chamber, with single-site endocardial or epicardial ventricular leads. Propensity to ventricular pacing depends on the initial indication for implant; bradycardia in ACHD may result from surgical trauma and/or scarring to the sinus or atrioventricular (AV) node, progressive atrial fibrosis, treatments for atrial arrhythmia or an anatomical abnormality 
of the conduction system. ${ }^{7}$ Concerningly, patients with ACHD may be more vulnerable to the adverse effects of ventricular pacing, since they also have multiple predisposing risk factors for heart failure. ${ }^{8}$ Certain congenital lesions, such as those with a systemic right ventricle, have a predisposition to ventricular failure ${ }^{9}$; differentiating this from pacing-induced cardiomyopathy is challenging. Nevertheless, identification of 'at-risk' individuals is valuable, as cardiac resynchronisation therapy (CRT) is an effective intervention in both acquired heart disease and ACHD ${ }^{10-12}$ We sought to determine the incidence, predictors and outcomes of pacing-associated cardiomyopathy in a population with ACHD.

\section{METHODS}

\section{Study population and clinical characteristics}

We retrospectively identified all patients with ACHD in our database with a permanent pacemaker (PPM), who had been seen at least two times in our unit between 2000 and 2019. Our database represents a quaternary referral centre with active follow-up of over 2000 patients with ACHD. Patients with congenital CHB and a structurally normal heart were excluded, as were patients with an implantable defibrillator or a de novo CRT implant. Clinical history was extracted from the medical records, with investigations taken from the most recent visit prior to PPM implantation. Systemic (subaortic) ventricular systolic function was measured using Simpson's rule for subaortic left ventricles and subjective grading for subaortic right ventricles. Mild dysfunction was defined as an ejection fraction of $40 \%-49 \%$, moderate $30 \%-39 \%$ and severe $<30 \%$. Complexity of CHD was classified according to the Bethesda criteria. ${ }^{13}$ Patients were not involved in this research.

\section{Pacing-induced cardiomyopathy}

The indication for PPM implant was categorised as sinus node disease, AV block, post-surgical sinus node disease, post-surgical AV block, tachy-brady syndrome or other. Patients were then followed for the primary endpoint of pacing-associated cardiomyopathy (PACM). This was defined as a decline in ejection fraction of at least one grade ( $\geq 10 \%$ if subaortic $\mathrm{LV}$, or subjective assessment if subaortic right ventricle), to a value $<50 \%$, in the absence of another identifiable aetiology (eg, valvular heart disease). Given patients with ACHD may develop ventricular impairment due to the natural progression of their disease, the 'PACM' endpoint was comprised of cardiomyopathy deemed 'possibly' or 'probably' related to ventricular pacing, as assessed by an ACHD cardiologist and electrophysiologist. The secondary endpoint was heart failure admission for any cause. Cardiomyopathy was chosen as the primary endpoint, rather than heart failure admission, as these were not patients with heart failure per se, and PACM may develop in the absence of symptoms; ventricular impairment is associated with adverse clinical outcomes in the setting of complex
CHD. ${ }^{8}{ }^{14}$ Prespecified subanalysis was performed stratifying patients into two groups based on age at PPM implant: PPM implant $<18$ years old vs $\geq 18$ years old. This cut-off was chosen to further assess long-term outcomes of paediatric versus adult pacing. Ventricular pacing percentage (VP\%) was recorded at first review post-PPM implant and at last review. VP\% at last review was recorded immediately prior to the development of PACM, or at last follow-up for those who did not develop this endpoint. $\mathrm{VP} \%$ at last review was used for the purposes of predictive analysis. In the patients who underwent upgrade to CRT, a 'non-responder' was defined as those with no improvement in ejection fraction, or a decline in ejection fraction, post-CRT.

\section{Statistical analysis}

Continuous variables are presented as mean $\pm \mathrm{SD}$, or median $\pm \mathrm{IQR}$. Categorical variables are presented as frequencies with percentages. A receiver-operating characteristic (ROC) curve was constructed to assess the optimal cut-off for VP\% that best predicted the PACM endpoint. Survival free from PACM was assessed by the Kaplan-Meier method. Variables associated with PACM or heart failure admission were assessed by the $\mathrm{X}^{2}$, Fisher exact or t-test as appropriate, on univariate analysis. Variables assessed were as follows: age, gender, systemic right ventricle, complex CHD, prior heart failure episode, QRS width, QRS morphology (leftbundle branch block), preexisting systemic ventricular impairment and VP\%. Multivariate logistic regression models were performed to identify independent predictors of PACM, incorporating the following variables added sequentially: demographics (age and gender), systemic ventricular morphology (left, right or univentricular), $\mathrm{VP} \%$, duration of pacing. A twosided $p$ value of $<0.05$ was considered statistically significant. Statistical analysis was performed using SPSS V.22.0.

\section{RESULTS}

\section{Study population}

Of 2073 patients in our database, we identified 106 patients with a PPM, who were seen at least two times in our unit between 2000 and 2019 (5.1\%). The median age at implant was 29 years (IQR 16-40), with a median follow-up post-implant of 9.4 years (IQR 4.0-21.5). Thirty-four patients were less than 18 years old at time of implant (32\%; median age 9), and 72 patients were greater than 18 years old (68\%; median age 36). CHD diagnoses are displayed in figure 1 . Baseline characteristics of the PPM patients are shown in table 1. Of 55 patients with a history of supraventricular arrhythmia, $35 \%$ had previously undergone catheter ablation; the AV node was intentionally ablated in one patient.

\section{Indications and implants}

PPM implantation was for sinus node disease in 29 (27\%), tachy-brady syndrome in $13(12 \%)$, AV block in $34(32 \%)$, post-surgical sinus node disease in $3(3 \%)$, post-surgical AV block in $20(19 \%)$ and other reasons 


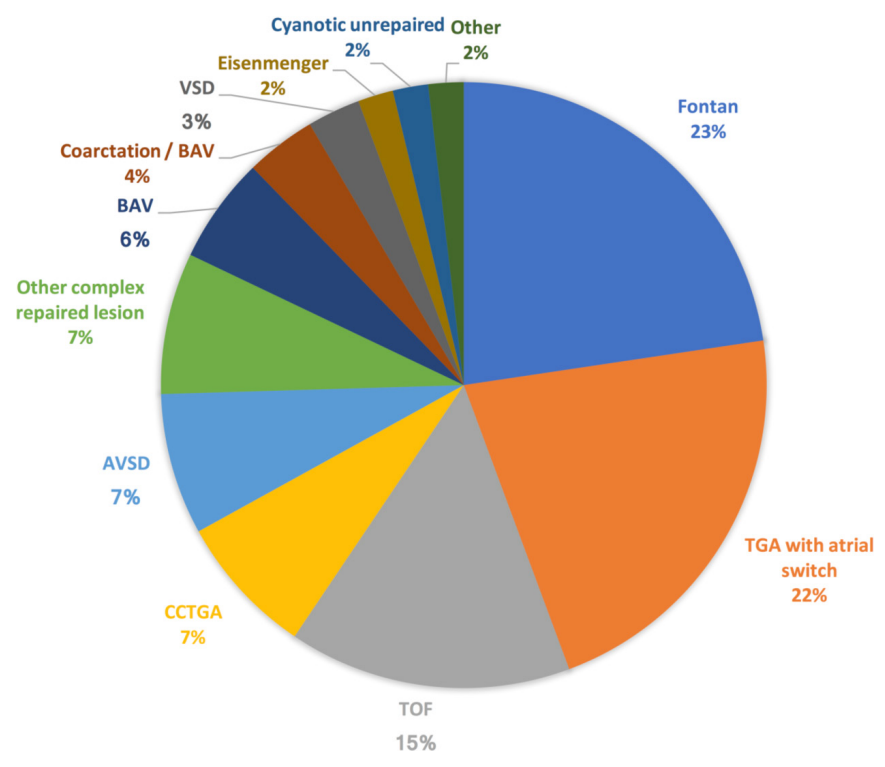

Figure 1 Congenital heart disease diagnoses. AVSD, atrioventricular septal defect; BAV, bicuspid aortic valve; CCTGA, congenitally corrected transposition of the great arteries; TGA, transposition of the great arteries; TOF, tetralogy of Fallot; VSD, ventricular septal defect.

(for example, syncope with suspected bradycardia) in $7(7 \%)$. The total proportion of pacemakers implanted for AV block was $51 \%$. Devices were dual chamber in 71 patients $(67 \%)$, atrial lead only in $3(3 \%)$ and ventricular lead only in $31(30 \%)$. Ten of the single chamber devices were subsequently upgraded to dual chamber (one atrial, nine ventricular). Atrial leads were endocardial in $65 \%$ and epicardial in $35 \%$. Ventricular leads were endocardial in $51 \%$ and epicardial in $49 \%$. Early complications (within 30 days of implant) occurred in 13 patients $(12 \%)$ and late complications in 29 patients (27\%). Early complications included lead revision in five patients, pocket haematoma in three, pneumothorax in three, tamponade in one and early death in one. Late complications included lead revision/failure in 29 patients, endocarditis in 3 and allergy to device component in 1 (noting several patients suffered more than one complication). Mean VP\% at first review post-implant was $51 \% \pm 43 \%$ and at last review was $54 \% \pm 44 \%$; these were highly correlated $(\mathrm{p}<0.001)$. In those paced for 'AV block', mean VP\% was $83 \%$, and in all others mean VP\% was $25 \%(\mathrm{p}<0.001)$.

\section{Pacing-associated cardiomyopathy}

The primary endpoint of PACM occurred in 25 patients $(24 \%)$, at a median time of 11.7 years post-implant (IQR 2.5-23.1). PACM was deemed 'probably' due to ventricular pacing in 19 patients and 'possibly' related in 6 patients. The primary endpoint, as a proportion of each CHD diagnosis, occurred as follows: transposition of the great arteries (TGA-atrial switch and congenitally corrected) in 10/31 patients (32\%), Fontan circulation in 2/24 patients (8\%), other complex CHD in 4/12 (33\%), tetralogy of Fallot (TOF) in 5/16 (31\%) and other simplemoderate CHD in 4/23 (17\%). PACM occurred in 10/34

\begin{tabular}{|c|c|}
\hline Characteristic & \\
\hline Male gender $(\mathrm{n}, \%)$ & $53(50)$ \\
\hline History of supraventricular arrhythmia (n, \%) & $55(52)$ \\
\hline Prior CCF admission (n, \%) & $8(8)$ \\
\hline \multicolumn{2}{|l|}{ Ventricular morphology (n, \%) } \\
\hline Systemic LV & 40 (38) \\
\hline Systemic RV & $40(38)$ \\
\hline Single ventricle & $26(25)$ \\
\hline \multicolumn{2}{|l|}{ Systemic ventricle impairment (n, \%) } \\
\hline Normal & $67(63)$ \\
\hline Mild & $32(30)$ \\
\hline Moderate & $7(7)$ \\
\hline \multicolumn{2}{|l|}{ Subpulmonary ventricle impairment (n, \%) } \\
\hline Normal & $71(67)$ \\
\hline Mild & $8(8)$ \\
\hline Moderate & $1(1)$ \\
\hline $\mathrm{N} / \mathrm{A}$ & $26(25)$ \\
\hline Moderate-severe systemic AVV regurgitation (n, \%) & $6(6)$ \\
\hline QRS width (ms, mean \pm SD) & $123 \pm 28$ \\
\hline \multicolumn{2}{|l|}{ QRS morphology (n, \%) } \\
\hline RBBB & $36(34)$ \\
\hline LBBB & $4(4)$ \\
\hline IVCD & $19(18)$ \\
\hline \multicolumn{2}{|l|}{ Ventricular lead route $(\mathrm{n}, \%)^{*}$} \\
\hline Endocardial & $53(51)$ \\
\hline Epicardial & $51(49)$ \\
\hline
\end{tabular}

${ }^{*}$ Two patients had atrial leads only.

AVV, atrioventricular valve; CCF, congestive cardiac failure; IVCD, interventricular conduction delay; LBBB, left bundle branch block; LV, left ventricle; N/A, not available; RBBB, right bundle branch block; RV, right ventricle.

patients with PPM implant at age $<18$ years old $(29 \%)$ and $15 / 72$ patients with PPM implant at age $\geq 18$ years old $(21 \%)$. The mean time to PACM was significantly longer in those paced from age $<18$ years old versus those paced aged $\geq 18$ years old (mean $24 \pm 12$ years vs $4 \pm 4$ years postimplant, respectively, $\mathrm{p}<0.001)$. Congenital diagnoses were evenly distributed between the two age groups. A deterioration in ejection fraction with a defined cause, such as valvular heart disease, occurred in 7/81 patients not meeting the primary endpoint $(9 \%)$. Heart failure admission for any cause occurred in 24 patients (23\%), at a median time of 5.7 years post-implant. Patients with PACM were more likely to be admitted with heart failure, compared with those who did not develop PACM (44\% vs $15 \%, \mathrm{p}=0.002$ ).

\section{Predictors of PACM: ventricular-pacing burden}

On univariate analysis, higher $\mathrm{VP} \%$ alone was predictive of PACM ( $p<0.001)$, as a continuous variable. Specifically, 


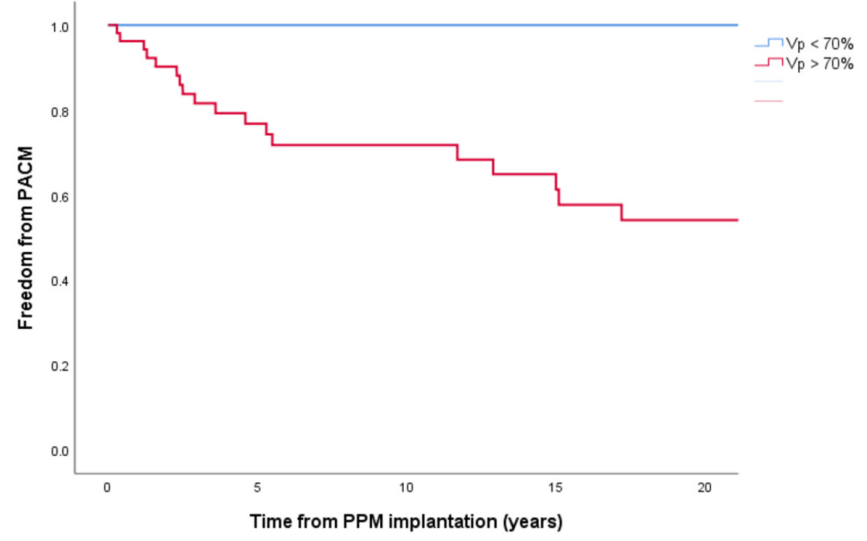

Number at risk

$\begin{array}{llllll}\text { VP }<70 \% & 53 & 40 & 25 & 20 & 15 \\ V P \geq 70 \% & 53 & 31 & 22 & 18 & 14\end{array}$

Figure 2 Survival free from pacing-associated cardiomyopathy (PACM). PPM, permanent pacemaker; VP, ventricular pacing.

pre-existing systemic ventricular impairment was not associated with PACM. Multivariate logistic regression models are presented in online supplemental figure S1. From ROC curve analysis, the optimal VP\% for predicting PACM was VP $\geq 70 \%$. Positive and negative predictive values, sensitivity, specificity of $\mathrm{VP} \geq 70 \%$, along with the ROC curve, is provided in online supplemental figure S2. Of the 53 patients with VP $<70 \%$, 0 developed the endpoint of PACM and of 53 patients with VP $\geq 70 \%, 25$ developed the endpoint ( $0 \%$ vs $47 \%, \mathrm{p}<0.001)$. Survival free from PACM is shown in figure 2. The development of PACM was analysed according to select CHD lesions and is shown in table 2 . VP $\geq 70 \%$ remained a significant predictor of PACM in TGA, TOF and complex biventricular repair subgroups of CHD. Fontan patients were more often paced for sick sinus syndrome, with a smaller proportion pacing $\geq 70 \%$ in the ventricle. When PACM did occur in a Fontan patient, ventricular-pacing burden was high. VP $\geq 70 \%$ remained significantly associated with PACM in those $<18$ years old at time of PPM implant and

Table 2 Analysis of pacing-associated cardiomyopathy (PACM) endpoint stratified by congenital heart disease lesion and ventricular pacing percentage (VP\%)

\begin{tabular}{lllcl}
\hline & & \multicolumn{2}{l}{ PACM endpoint } & \\
\cline { 3 - 4 } CHD lesion & $\begin{array}{l}\text { Mean } \\
\text { VP (\%) }\end{array}$ & $\begin{array}{l}\text { PACM } \\
\text { endpoint } \\
\text { VP }<70 \%\end{array}$ & VP $\geq 70 \%$ & P value \\
\hline TOF & 58 & $0 / 7(0 \%)$ & $5 / 9(56 \%)$ & 0.034 \\
\hline TGA $^{*}$ & 57 & $0 / 14(0 \%)$ & $10 / 17(59 \%)$ & 0.004 \\
Fontan & 34 & $0 / 17(0 \%)$ & $2 / 7(29 \%)$ & 0.130 \\
\hline Complex repair & 62 & $0 / 3(0 \%)$ & $4 / 5(80 \%)$ & 0.048 \\
\hline
\end{tabular}

${ }^{*}$ Atrial switch and congenitally corrected, that is, those with systemic right ventricles.

CHD, congenital heart disease; TGA, transposition of the great arteries; TOF, tetralogy of Fallot. those $\geq 18$ years old, analysed independently. Post-hoc analysis excluding all 'possible' patients with PACM (ie, including only 'probable' PACM) did not change the significant association of VP $\geq 70 \%$ with PACM. Ventricular lead pacing site did not significantly affect rates of PACM (15/53 endocardial vs $10 / 51$ epicardial leads developed PACM; $28 \%$ vs $20 \%$; $\mathrm{p}=0.300$ ). There was no significant difference in mean $\mathrm{VP} \%$ between those patients with endocardial versus epicardial leads $(55 \%$ vs $48 \% ; \mathrm{p}=0.154)$. There were no significant predictors of 'heart failure admission for any cause'.

\section{Outcomes in patients with PACM}

The interventions and outcomes in the 25 patients who met the primary endpoint are shown in table 3. A total of 11 patients were upgraded to CRT pacemaker \pm defibrillator, with 9 responders and 2 non-responders (ie, responder rate $82 \%$ ). CRT upgrade is planned, but not yet performed, in a further three patients. In the CRT upgrades, systemic ventricular leads were implanted endocardially via the coronary sinus in six patients (four TOF, one congenitally corrected TGA, one repaired truncus arteriosus), endocardially via a persistent leftsided superior vena cava in one patient (repaired ventricular septal defect), with surgical epicardial leads in four patients (TGA post-Mustard, congenitally corrected TGA, Ebstein's anomaly, aortic stenosis). A total of 11 patients from the entire cohort were upgraded to an implantable cardioverter-defibrillator, without CRT capability (10\%). There were 14 deaths (13\%) during follow-up, at a mean time of 11.4 years post-PPM implant (range 0.1-27.8 years). Deaths were due to heart failure $(n=8)$, infective endocarditis $(n=2)$, pulmonary hypertension/respiratory failure $(\mathrm{n}=2)$ and cerebrovascular events $(n=2)$. In the group who met the primary endpoint, four deaths occurred: one due to stroke and three due to heart failure (one Mustard patient who failed to respond to CRT, as well as Fontan and Rastelli patients who were too unwell for intervention).

\section{DISCUSSION}

In a cohort of patients with ACHD followed long-term post-pacemaker implantation, one in four developed an otherwise unexplained decline in systemic ventricular function, significantly associated with a high burden of ventricular pacing ( $\mathrm{VP} \geq 70 \%$ ). The adverse effects of long-term single-site ventricular pacing have been appreciated for some time in the population with acquired heart disease. ${ }^{1-4}$ Ventricular pacing results in electrical and mechanical dyssynchrony of the systemic ventricle, reducing effective stroke volume. ${ }^{5}$ At a histopathological level, myofibrillar disarray has been observed in a canine-pacing model and degenerative fibrosis in biopsies from chronically paced young patients with congenital CHB. ${ }^{615}$ Despite this, a subset of patients appear to tolerate long-term ventricular pacing well; risk factors for pacing-induced cardiomyopathy in acquired heart disease 
Table 3 Interventions and outcomes in patients who developed pacing-associated cardiomyopathy

\begin{tabular}{|c|c|c|c|}
\hline CHD diagnosis & $\mathbf{N}$ & Intervention/outcome & Response to CRT \\
\hline \multirow[t]{3}{*}{ TOF } & 5 & CRT upgrade $(n=3)$ & $3 / 3$ responded \\
\hline & & CRT upgrade intended $(n=1)$ & \\
\hline & & Lost to follow-up $(n=1)$ & \\
\hline \multirow[t]{6}{*}{$\mathrm{TGA}^{*}$} & 10 & CRT upgrade $(n=4)$ & $3 / 4$ responded \\
\hline & & CRT upgrade intended $(n=1)$ & \\
\hline & & Medical therapy only $(n=2)$ & \\
\hline & & Listed for heart/lung transplant $(n=1)$ & \\
\hline & & Death $(n=1)$ & \\
\hline & & Lost to follow-up $(n=1)$ & \\
\hline \multirow[t]{2}{*}{ Fontan } & 2 & Medical therapy only $(n=1)$ & \\
\hline & & Death $(n=1)$ & \\
\hline \multirow[t]{3}{*}{ Other complex CHD } & 4 & CRT upgrade $(n=1)$ & $1 / 1$ responded \\
\hline & & Improved with reduction in VP\% $(n=1)$ & \\
\hline & & Medical therapy only $(n=2)$ & \\
\hline Other simple-moderate CHD & 4 & $\begin{array}{l}\text { CRT upgrade }(n=3) \\
\text { CRT upgrade intended }(n=1)\end{array}$ & $2 / 3$ responded \\
\hline
\end{tabular}

${ }^{*}$ Atrial switch and congenitally corrected, that is, those with systemic right ventricles.

$\mathrm{CHD}$, congenital heart disease; CRT, cardiac resynchronisation therapy; TGA, transposition of the great arteries; TOF, tetralogy of Fallot; VP\%, ventricular pacing percentage.

include pre-existing systemic ventricular impairment and a higher burden of ventricular pacing. ${ }^{1-4}$ Patients with ACHD may be especially susceptible to this phenomenon, as this population has multiple predisposing risk factors for heart failure, depending on the underlying lesion. ${ }^{89}$

In acquired heart disease, approximately 10\%-20\% of patients will develop systemic ventricular dysfunction when followed long-term post-pacing. ${ }^{1-4}$ Our incidence of $24 \%$ exceeds these reports, but may overestimate true 'pacing-induced cardiomyopathy' due to the natural history of systemic ventricular decline in certain congenital lesions, such as those with systemic right ventricles. In patients with ACHD it is inherently difficult to delineate the relative contribution of pacing from natural disease progression particularly in those with pre-existing ventricular impairment prior to pacing, or from cardiomyopathy related to surgical intervention. Interestingly, in our study those patients paced in adulthood typically developed PACM in the short midterm (mean 4 years) and those paced in childhood typically developed PACM very late post-implant (mean 24 years). This may suggest that a significant proportion of patients with ACHD paced in childhood developed ventricular decline due to natural disease progression, rather than pacing. Pacing-induced cardiomyopathy in acquired heart disease cohorts usually occurs in the first 4 years, ${ }^{1-4}$ however, in long-term follow-up of children paced for congenital CHB, up to $10 \%$ developed LV impairment at a mean time of 15 years post-implant. ${ }^{16}$ It is possible that a minority of vulnerablepaced young patients may suffer very late cardiomyopathy, despite many years of stable ventricular function and thus lifelong monitoring of ventricular function is warranted. ${ }^{17}$ Pacing dyssynchrony may also exacerbate late systemic ventricular decline due to natural disease progression in ACHD. Incidence of PACM in 'whole of ACHD' cohorts has not previously been assessed. The relatively low rates of systemic ventricular decline due to a specific (non-PACM) aetiologies may reflect the young average age of our population.

The development of PACM was significantly associated with a high burden of ventricular pacing ( $\mathrm{VP} \geq 70 \%)$ in our study. This 'exposure-response' relationship further supports the pathophysiological link between chronic dyssynchrony and ventricular impairment. Importantly, this association does not prove causation, as patients with more extensive myocardial and/or electrical disease are more likely to require pacing. Previously, small singlelesion studies in ACHD have supported a correlation between moderate-high burden ventricular pacing and cardiomyopathy in univentricular hearts and congenitally corrected TGA, but failed to show this in TOF. ${ }^{18-20}$ In our study, the correlation between high-burden pacing and cardiomyopathy was consistent across TGA (atrial switch and congenitally corrected), TOF and complex biventricular repair subgroups. Conversely, most Fontan patients were paced for sick sinus syndrome and thus both highburden ventricular pacing and PACM were uncommon. The preferred 'cut-off' in VP\% that best predicts cardiomyopathy is contentious. In the MOST trial, a VP $>40 \%$ was predictive of heart failure hospitalisation. ${ }^{1}$ This cut-off was then adopted and supported by several subsequent studies, although cardiomyopathy with VP $>20 \%$ has also clearly been described. ${ }^{23}$ The proportion of patients with ventricular pacing between $20 \%$ and $70 \%$ in our study 
was relatively small, and so our study may not have been powered to detect this association. We suggest vigilance is required in any patient with ACHD expected to have even a modest burden of ventricular pacing. Notably, in contrast to acquired heart disease, pre-existing systemic ventricular impairment was not associated with the development of PACM. Those patients who developed PACM were more likely to be admitted with heart failure in our study, but VP\% per se was not predictive of 'heart failure admission'. This may be due to the multiple heterogeneous aetiologies driving heart failure admission in ACHD, diluting the significance of ventricular pacing alone.

In patients exposed to unavoidable pacing with declining systemic ventricular function, upgrade to CRT should be considered. In those who were upgraded to CRT in our study, response rates were high, exceeding $80 \%$. This supports the ACHD literature, which has reported CRT response rates of $80 \%-90 \%$, favourable in comparison with acquired heart disease. ${ }^{10-12}$ Despite the anatomical complexities in ACHD, complication rates of CRT implant or upgrade were not prohibitive in these studies. ${ }^{11} 12$ The subgroup of patients with ACHD with PACM and upgrade may have a greater likelihood of response than de novo CRT implants, highlighting the importance of making this diagnosis. ${ }^{12}$ Nevertheless, uncertainties do remain regarding CRT response rates within select CHD lesions. It is unclear if multisite 'biventricular' pacing in the setting of a Fontan heart is clinically beneficial, with conflicting evidence. ${ }^{12} 21$ Systemic right ventricles are another area of contention, with one study finding a 'systemic LV' was associated with CRT responders, while another found equivalent response rates in systemic left or right ventricles. ${ }^{11}{ }^{12} \mathrm{~A}$ total of $11 / 25$ patients in our study who met the primary endpoint were not upgraded to CRT (or referred for pending CRT). The reasons for this were complex, but included non-ambulatory class IV New York Heart Association symptoms, death, loss to follow-up or a clinician decision to pursue medical therapy. Notably, the exclusion of more unwell patients from CRT upgrade will increase the proportion of CRT responders reported in our study.

There are several important clinical implications of our research. First, surveillance echocardiography is a simple and appropriate intervention in patients with ACHD exposed to ventricular pacing even at a modest burden. Given the promising response rates to CRT upgrade in patients with ACHD with PACM, our data suggest that this is an eminently treatable condition, and that CRT should be considered in patients with CHD who are ventricularly paced and have declining ventricular function. Second, algorithms to reduce VP\% may be beneficial. In acquired heart disease, these algorithms are effective at reducing VP\%, but extrapolation to positive clinical endpoints has been disappointing. ${ }^{22}$ 'Managed ventricular pacing' has been shown to reduce $\mathrm{VP} \%$ in $\mathrm{CHD},{ }^{23}$ but clinical endpoints of this intervention have not been assessed.
VP\% may also be reduced by reduction of pharmacological rate-slowing therapy, where appropriate. Finally, the selection of patients with CHD with an indication for pacing who would benefit from de novo CRT is an important area of future research. In acquired heart disease, guidelines suggest this should be considered in patients with heart failure and reduced ejection fraction, with an expected high burden of ventricular pacing (typically $>40 \%) .{ }^{24}$ This recommendation has been extrapolated to ACHD guidelines. ${ }^{7}$

\section{Limitations}

There are several limitations to our study. Our study is retrospective and single-centre. Prevalence of sinus node disease versus AV block varies across differential congenital lesions, thus skewing the proportion of CHD diagnoses in the VP $\geq 70 \%$ vs VP $<70 \%$ subgroups. Despite this, VP $\geq 70 \%$ remained significantly associated with PACM across multiple congenital lesions. Relative numbers of patients with VP\% between $20 \%$ and $70 \%$ were low, and so this study may not have been powered to detect an association with PACM in this moderate pacing group. The use of AV nodal blocking or antiarrhythmic drugs was not analysed, but these may affect VP\%. Differentiating pacing-induced cardiomyopathy from natural progression of disease is inherently difficult in ACHD, and thus our findings do not represent a true incidence of 'pacing-induced cardiomyopathy', but rather an association between ventricular pacing and the endpoint of deteriorating ventricular function. Clinical assessment is likely to overestimate 'true' pacing-induced cardiomyopathy incidence.

\section{CONCLUSIONS}

In a cohort of patients with ACHD followed longterm post-pacemaker implantation, a high burden of ventricular pacing $(\mathrm{VP} \geq 70 \%)$ was significantly associated with the development of cardiomyopathy. This association was independently present across TGA, TOF and complex biventricular repair lesion subgroups. Patients with ACHD expected to pace in the ventricle should be closely monitored for PACM, and affected patients be considered for upgrading to biventricular pacing.

Acknowledgements BM was supported by a Health Professional Scholarship from the National Heart Foundation of Australia.

Contributors BM was responsible for research design, acquisition and interpretation of data, drafting and critically revising the manuscript and approval of the final version. BM is responsible for the overall content as a guarantor. CM was responsible for research design, critically revising the manuscript and approval of the final version. MM was responsible for research design, critically revising the manuscript and approval of the final version. DSC was responsible for research design, critically revising the manuscript and approval of the final version. RLC was responsible for research design, critically revising the manuscript and approval of the final version.

Funding The authors have not declared a specific grant for this research from any funding agency in the public, commercial or not-for-profit sectors.

Competing interests None declared.

Patient consent for publication Not required. 
Ethics approval Ethics approval for this study was obtained from the Sydney Local Health District Human Research Ethics Committee, Camperdown, New South Wales, Australia (2019/STE16575).

Provenance and peer review Not commissioned; external peer review.

Data availability statement Deidentified participant data are available from BM (ORCID identifier 0000-0003-3645-0890), upon reasonable request.

Open access This is an open access article distributed in accordance with the Creative Commons Attribution Non Commercial (CC BY-NC 4.0) license, which permits others to distribute, remix, adapt, build upon this work non-commercially, and license their derivative works on different terms, provided the original work is properly cited, appropriate credit is given, any changes made indicated, and the use is non-commercial. See: http://creativecommons.org/licenses/by-nc/4.0/.

ORCID iD

Benjamin M Moore http://orcid.org/0000-0003-3645-0890

\section{REFERENCES}

1 Sweeney MO, Hellkamp AS, Ellenbogen KA, et al. Adverse effect of ventricular pacing on heart failure and atrial fibrillation among patients with normal baseline QRS duration in a clinical tria of pacemaker therapy for sinus node dysfunction. Circulation 2003:107:2932-7.

2 Sharma AD, Rizo-Patron C, Hallstrom AP, et al. Percent right ventricular pacing predicts outcomes in the DAVID trial. Heart Rhythm 2005;2:830-4.

3 Khurshid S, Epstein AE, Verdino RJ, et al. Incidence and predictors of right ventricular pacing-induced cardiomyopathy. Heart Rhythm 2014;11:1619-25.

4 Cho SW, Gwag HB, Hwang JK, et al. Clinical features, predictors, and long-term prognosis of pacing-induced cardiomyopathy. Eur $J$ Heart Fail 2019;21:643-51.

5 Tops LF, Schalij MJ, Bax JJ. The effects of right ventricular apical pacing on ventricular function and dyssynchrony implications for therapy. J Am Coll Cardiol 2009;54:764-76.

6 Karpawich PP, Rabah R, Haas JE. Altered cardiac histology following apical right ventricular pacing in patients with congenital atrioventricular block. Pacing Clin Electrophysiol 1999;22:1372-7.

7 Khairy P, Van Hare GF, Balaji S, et al. PACES/HRS expert consensus statement on the recognition and management of arrhythmias in adult congenital heart disease: developed in partnership between the pediatric and congenital electrophysiology Society (PACES) and the heart rhythm Society (HRS). endorsed by the governing bodies of PACES, Hrs, the American College of cardiology (ACC), the American heart association (AHA), the European heart rhythm association (EHRA), the Canadian heart rhythm Society (CHRS), and the International Society for adult congenital heart disease (ISACHD). Heart Rhythm 2014;11:e102-65.

8 Budts W, Roos-Hesselink J, Rädle-Hurst T, et al. Treatment of heart failure in adult congenital heart disease: a position paper of the
Working Group of Grown-Up Congenital Heart Disease and the Heart Failure Association of the European Society of Cardiology. Eur Heart J 2016;37:1419-27.

9 Duncan BW, Mee RBB. Management of the failing systemic right ventricle. Semin Thorac Cardiovasc Surg 2005;17:160-9.

10 Curtis AB, Worley SJ, Adamson PB, et al. Biventricular pacing for atrioventricular block and systolic dysfunction. N Engl J Med 2013;368:1585-93.

11 Dubin AM, Janousek J, Rhee E, et al. Resynchronization therapy in pediatric and congenital heart disease patients: an international multicenter study. J Am Coll Cardiol 2005;46:2277-83.

12 Janousek J, Gebauer RA, Abdul-Khaliq H, et al. Cardiac resynchronisation therapy in paediatric and congenital heart disease: differential effects in various anatomical and functional substrates. Heart 2009;95:1165-71.

13 Warnes CA, Liberthson R, Danielson GK, et al. Task force 1: the changing profile of congenital heart disease in adult life. J Am Coll Cardiol 2001;37:1170-5.

14 Valente AM, Gauvreau K, Assenza GE, et al. Contemporary predictors of death and sustained ventricular tachycardia in patients with repaired tetralogy of Fallot enrolled in the INDICATOR cohort. Heart 2014;100:247-53.

15 Adomian GE, Beazell J. Myofibrillar disarray produced in normal hearts by chronic electrical pacing. Am Heart $J$ 1986;112:79-83.

$16 \mathrm{Kim}$ JJ, Friedman RA, Eidem BW, et al. Ventricular function and longterm pacing in children with congenital complete atrioventricular block. J Cardiovasc Electrophysiol 2007;18:373-7.

17 Moak JP, Barron KS, Hougen TJ, et al. Congenital heart block: development of late-onset cardiomyopathy, a previously underappreciated sequela. J Am Coll Cardiol 2001;37:238-42.

18 Bulic A, Zimmerman FJ, Ceresnak SR, et al. Ventricular pacing in single ventricles-A bad combination. Heart Rhythm 2017;14:853-7.

19 Yeo WT, Jarman JWE, Li W, et al. Adverse impact of chronic subpulmonary left ventricular pacing on systemic right ventricular function in patients with congenitally corrected transposition of the great arteries. Int J Cardiol 2014;171:184-91.

20 Egbe AC, Najam M, Banala K, et al. Effect of right ventricular pacing on left ventricular systolic function in patients with tetralogy of Fallot. Int J Cardiol Heart Vasc 2020;26:100426.

21 O'Leary ET, Gauvreau K, Alexander ME, et al. Dual-Site ventricular pacing in patients with Fontan physiology and heart block: does it mitigate the detrimental effects of single-site ventricular pacing? JACC Clin Electrophysiol 2018;4:1289-97.

22 Wilkoff BL, Kudenchuk PJ, Buxton AE, et al. The DAVID (Dual Chamber and VVI Implantable Defibrillator) II trial. J Am Coll Cardiol 2009;53:872-80.

23 Kaltman JR, Ro PS, Zimmerman F, et al. Managed ventricular pacing in pediatric patients and patients with congenital heart disease. $A m J$ Cardiol 2008;102:875-8.

24 Normand C, Linde C, Singh J, et al. Indications for Cardiac Resynchronization Therapy: A Comparison of the Major International Guidelines. JACC Heart Fail 2018;6:308-16. 